\title{
Interspinous devices: are they as attractive as they seem? An intermediate-term follow-up
}

Authors Josh E Schroeder, Leon Kaplan, Shimon Barzilay, Yair Barzilay

Institution Spine Unit, Hadassah-Hebrew University Medical Center, Jerusalem, Israel

\begin{tabular}{l|l|}
\hline $\begin{array}{l}\text { Final Class of evidence } \\
\text { (CoE)-treatment }\end{array}$ & Yes \\
\hline Study design: \\
\hline RCT \\
\hline Cohort \\
\hline Case control \\
\hline Case series \\
\hline Methods \\
\hline Concealed allocation (RCT) \\
\hline Intention to treat (RCT) \\
\hline Blinded/independent \\
evaluation of primary outcome & \\
\hline F/U $\geq 85 \%$ & \\
\hline Adequate sample size & $\bullet$ \\
\hline Control for confounding & \\
\hline Overall class of evidence & IV \\
\hline
\end{tabular}

\section{ABSTRACT}

Study design: A retrospective cohort of 68 patients who underwent insertion of the DIAM (Medtronic Sofamor Danek, Switzerland) interspinous device (ISD) during 2006-2008 at one medical center.

Objectives: To assess the short- and intermediate-term outcomes and complications associated with ISD.

Methods: Evaluation of files and all patients who underwent insertion of a DIAM ISD was performed. Patients walking distances and pain (visual analog scale score) were compared with data gathered before surgery. Outcome and all complications related to ISD have been identified and analyzed.

Results: All 68 patients were available for follow-up. Mean follow-up was 34 months (23-52 months). The average age was $57( \pm 13)$ years. Walking distance increased by $890 \%$ and patient's pain score improved by 3.27 points on visual analog scale. Twenty-one (32\%) of the 68 patients had perioperative or late complications. Nine complications $(75 \%)$ were unrelated to ISD and included 5 dura tears, 3 woundrelated complications, and 1 transient ischemic attack. Spinous process fractures occurred in 5 cases, leading to revision in 2 cases. In total, 7 of the patients required revision surgery. These patients were older, with an average age of 69 years.

Conclusion: The outcome of patients who had an implantation of the DIAM ISD is good. In this cohort, 6\% developed recurrent claudication symptoms in the second postoperative year. In an older population, the combination of softer bone and rigid stenosis increase the risk of spinous process fracture, resulting in failure and leading to revision surgery. Other solutions should be sought for these patients.

The authors have no conflict of interest with this article.

No funding was received for this research study. 


\section{STUDY RATIONALE}

Interspinous devices (ISDs) are motion preservation systems that are claimed to alter favorably the movement and load transfer of a spinal-motion segment and to increase the space in the lateral recess and foramina [1-5]. Initial clinical results were promising $[4,5]$; however, in recent studies mixed results have been reported [1,2].

\section{OBJECTIVES}

To assess the outcome and complication rate of a cohort of patients who underwent a DIAM ISD implantation (Medtronic Sofamor Danek, Switzerland) in the short and intermediate postoperative periods.

\section{METHODS}

Study design: Retrospective cohort of all patients followed-up for 2 years or more after surgery.

Inclusion criteria: All patients with spinal claudication or radiculopathy, caused by spinal stenosis who were implanted with the DIAM ISD (Medtronic Sofamor Danek, Switzerland) during 2006-2008.

Exclusion criteria: Patients with insertion of other ISDs (Fig 1). Other ISDs were excluded as a mean to reduce variability, as the insertion process is different for each ISD and the methods of fixation are also different.

\section{Patient population (Fig 1):}

- Sixty-eight patients with spinal claudication or radiculopathy due to spinal stenosis who underwent insertion of a DIAM ISD (total 91 ISDs) were included in the study.
- Patients' comorbidities were documented (ie, hypertension, osteoporosis, diabetes mellitus, and so on).

\section{Interventions:}

- The ISD was implanted in 1 level for 52 patients; 2 levels for 16 patients; and 3 levels for 1 patient.

- Forty-eight patients underwent an insertion of ISD following a decompression procedure (laminotomy, flavectomy, recess decompression, or foraminotomy). The ISD was implanted according to published guidelines [6].

Outcomes: Patients walking time (in minutes) before and after the procedure was assessed (patients were asked "How long can you walk until your back and leg symptoms make you stop or sit down?").

- Patient back and leg pain was assessed via a 0-10 cm visual analog scale (VAS) before and after surgery.

- Placement of ISD was verified with a postoperative standing AP and lateral x-rays.

- All short- and intermediate-term complications were documented.

- Revision spine surgery was also documented.

\section{Analysis:}

- Descriptive statistical methods were used on the Microsoft Excel ${ }^{\circledR}$ spreadsheet software for counts, rates, means, and standard deviations of demographic and disease-related data.

- A paired $t$ test was used for comparing preoperative and postoperative VAS scores and walking distance; $P<.05$ was considered significant. For VAS scores, improvements beyond accepted minimal clinically important difference (1.8 points) were considered significant [7].

- Regression analysis was performed to find any comorbidity as a predictor of failure.

- All complications were noted. 
Fig 1 Patient sampling and selection. ISD indicates interspinous device; DIAM implant from Medtronic Sofamor Danek, Switzerland.

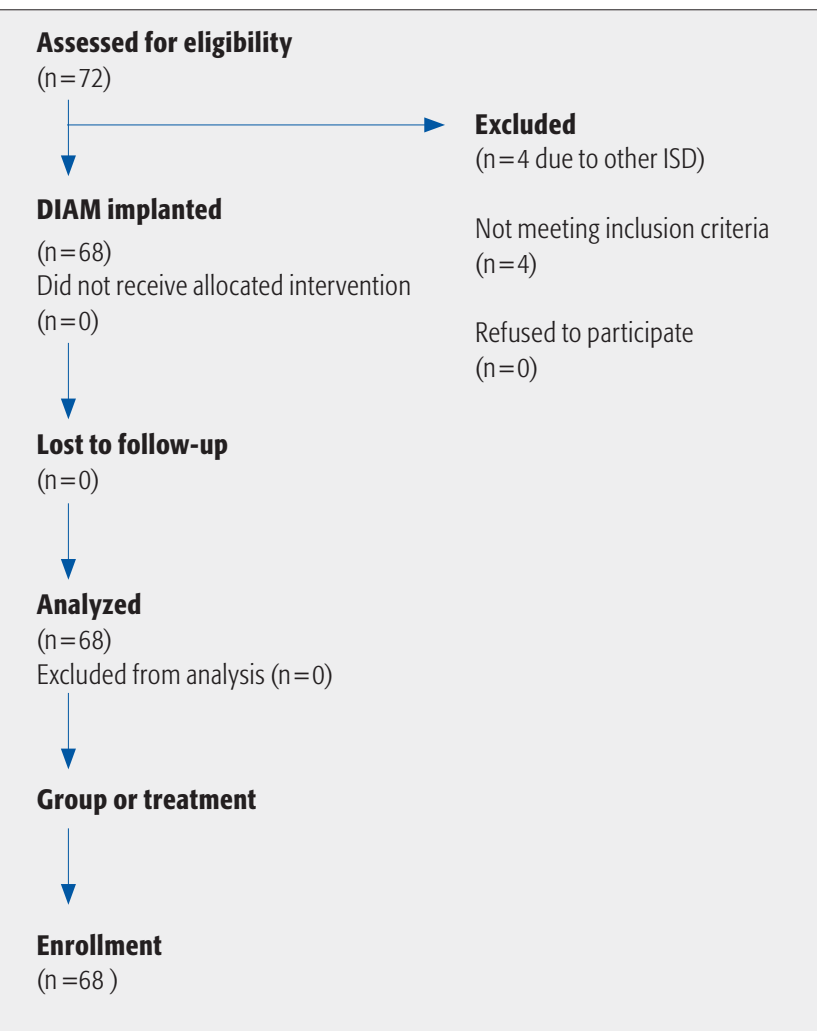

Table 1 Demographic and baseline characteristics of procedure.

\begin{tabular}{ll}
\hline & $\mathbf{N}=\mathbf{6 8}$ \\
\hline Age, $y$, mean \pm SD & $57 \pm 13$ \\
\hline Female & $34(41 \%)$ \\
\hline Decompression and ISD & $48(70.5 \%)$ \\
\hline ISD 1 level & $52(76.4 \%)$ \\
\hline ISD 2 levels & $15(22 \%)$ \\
\hline ISD 3 levels & 1 \\
\hline Spinal pathology & \\
\hline - Radiculopathy & 12 \\
\hline - Claudication & 56 \\
\hline Comorbidities & \\
\hline - Hypertension & 22 \\
\hline - Ischemic heart disease & 14 \\
\hline - Diabetes mellitus & 10 \\
\hline - Hypothyroidism & 2 \\
\hline - Asthma & 2 \\
\hline Other & 3 \\
\hline
\end{tabular}

Table 2 Time from surgery to complication.

\begin{tabular}{lll}
\hline Complication & No. of patients & Percentage \\
\hline Dural tear & 7 & 10.92 \\
\hline Fracture of spinous process & 6 & 8.82 \\
\hline Wound infection & 3 & 4.4 \\
\hline Revision & 7 & 10.92 \\
\hline Other complication & 3 & 4.4 \\
\hline
\end{tabular}

Table 3 Complications in relevant interspinous device studies.

\begin{tabular}{llll}
\hline Study & No. of patient & Overall complication rate, \% & Spinous process fracture rate, \% \\
\hline Current study & 68 & 24 & 7.3 \\
\hline Kondrashov et al [8] & 18 & 22 & Not reported \\
\hline Zucherman et al [5] 2005 & 100 & 11 & 1 \\
\hline Bowers et al [2] 2010 & 13 & 38 & 23 \\
\hline Barbagallo et al [1] 2009 & 69 & 11.6 & 6 \\
\hline
\end{tabular}


Fig 2a-b X-rays of AP lumbar spine of a 78-year-old man before surgery. Severe degenerative changes are seen and instability at the L3/L4 intervertebral disc (2b).
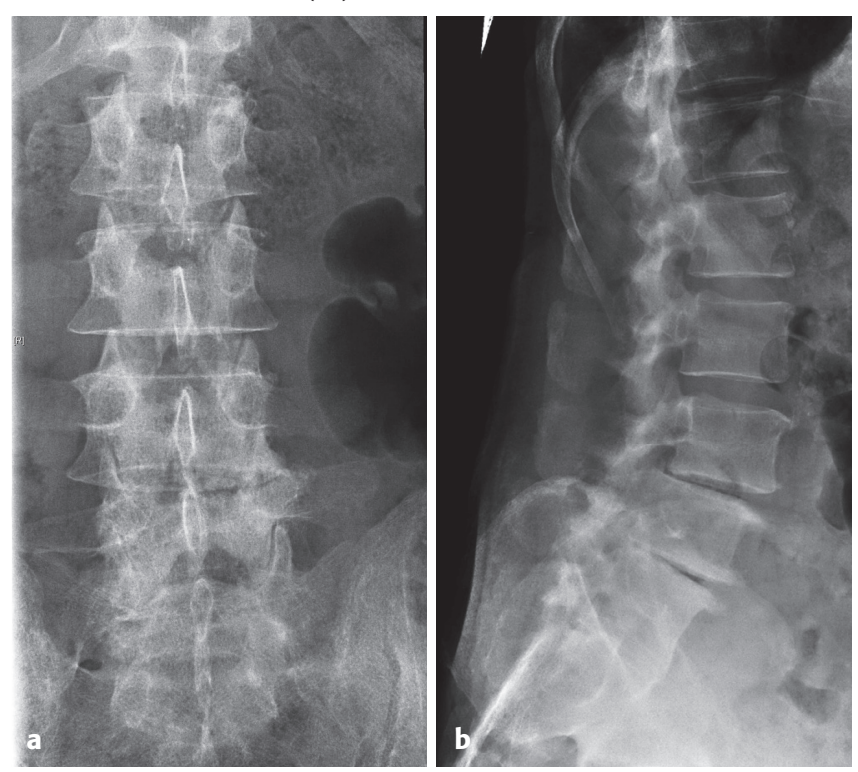

Fig 3b-c Axial view of the fracture height.
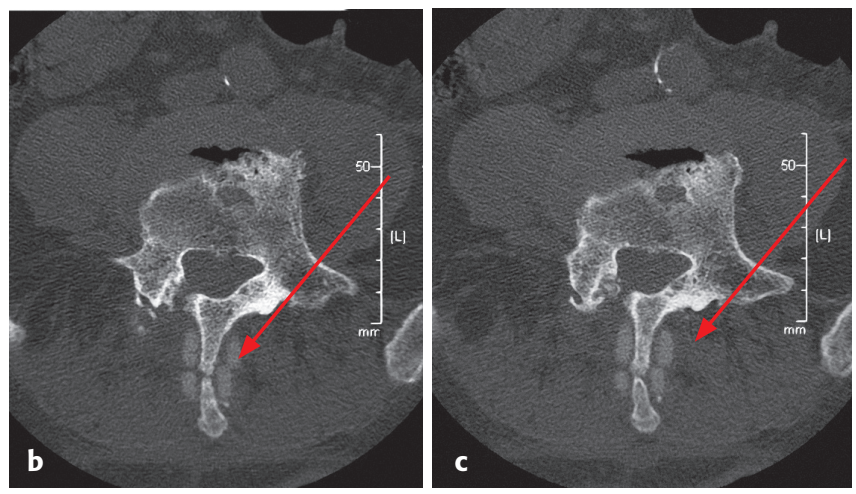

Fig 5a X-ray of AP lumbar spine of the patient after a revision surgery; he had the DIAMs removed and underwent formal transforaminal lumbar interbody fusion L3-L5. His symptoms subsided.

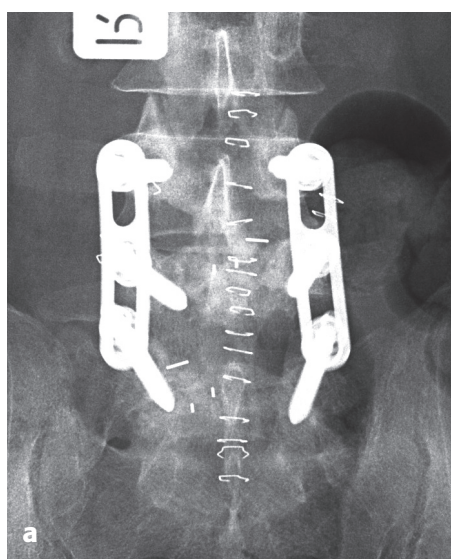

Fig 3a A sagittal postoperative computed tomographic scan of the same patient indicating a fracture of the $L 4$ spinous process between two DIAMs that were placed in the L3/4 and L4/5. Arrow indicates the fracture (the patient underwent decompression of these heights as well).

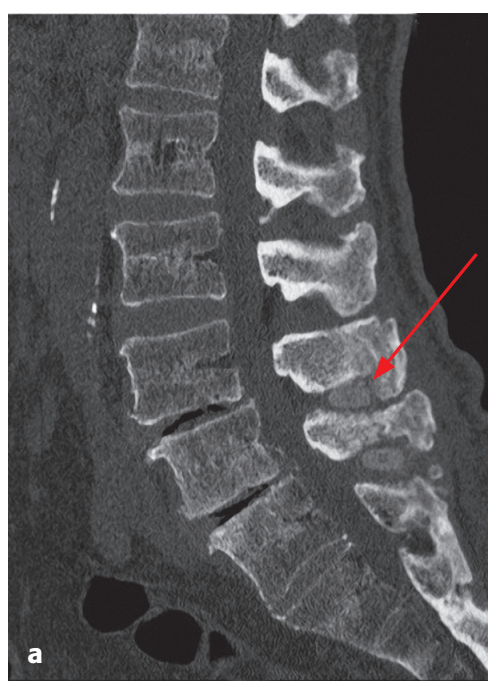

Fig 4a-b Sagittal views of a magnetic resonance imaging showing recurrent spinal stenosis (arrows) after the fracture.

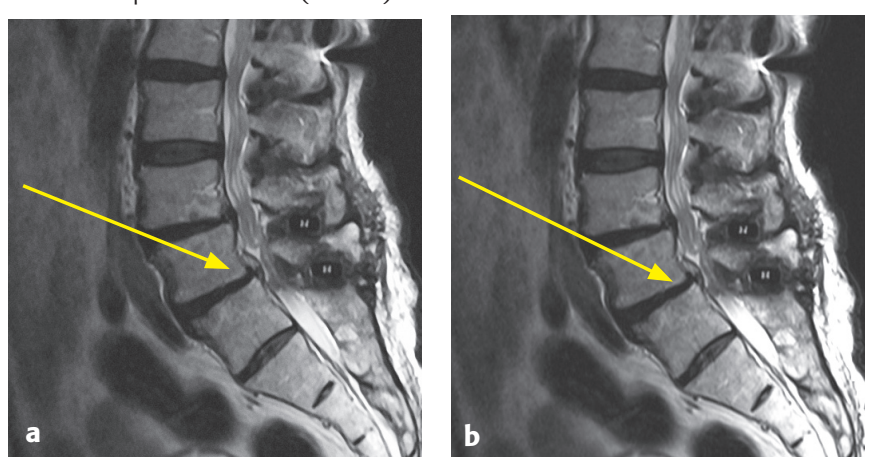

Fig 5b Lateral view of the lumbar spine of the same patient after transforaminal lumbar interbody fusion L3-L5.

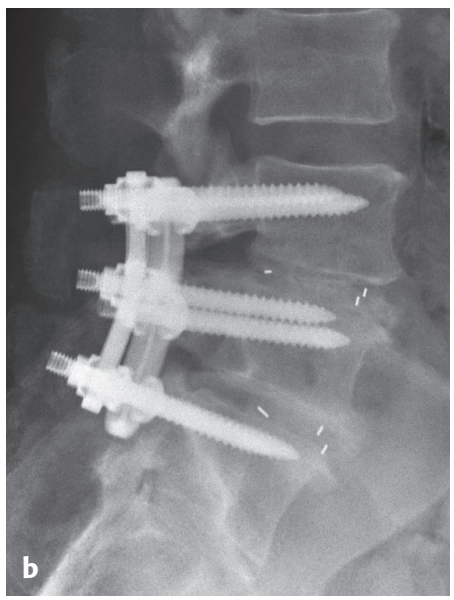




\section{RESULTS}

- Patients' demographics and surgical procedures are listed in Table 1.

- Mean age was 57 years (range, 43-75 years).

- In 20 cases ISD was implanted without decompression.

- Average follow-up was 34 months (range, 23-52 months).

- In all but one case, ISD was placed in the proper position.

- Mean VAS back pain score decreased from 6.14 before the procedure to 2.87 after the procedure $(P<.05$, and significant according to MCID values), and VAS leg score decreased from 8.9 to 1.45 after the procedure $(P<.05$, and significant according to MCID values).

- Mean walking improved from 14.1 minutes before surgery to 125.77 minutes after surgery $(P<.05)$.

- Of 68 patients implanted with interspinous devices, $21(32 \%)$ had complications (Figs 2-4).

- Nine complications (75\%) were unrelated to ISD and 12 were directly related to ISD (Table 2).

- Seven patients (10.1\%) underwent revision surgery (Fig 5).

- The average time to revision surgery was 21.6 months (range, 6-38 months).

- The average age of patients who suffered from ISD-related spinous process (SP) fractures was higher (69 years).

- Regression analysis did not find any significance in any comorbidity toward failure of DIAM.

\section{DISCUSSION}

- After 2.5 years, two-third of the patients who had surgery reported pain reduction and improved walking time. This benefit is similar to that seen in decompression surgery of spinal stenosis.

- Complications related to the decompression part of the procedure and wound infections were similar to other published data [9].

- Spinous process fractures were the most common complication related to ISD $(7.5 \%)$.

- At maximal follow-up, $40 \%$ of patients with SP fractures underwent revision surgery, compared with $10 \%$ in the whole series and $8 \%$ who did not have a fracture of the SP.

- The SP fractures occurred in older patients, and were most probably related to weaker bone and stiffer motion segments, reflecting an incorrect patient selection for the insertion of DIAM ISD.

- The distraction of SPs during DIAM implantation may have caused the fractures.

- In the second- and third-year follow-up six patients developed recurrent claudication symptoms, most should not have been implanted with ISD.

- The complication rate in other studies varies from $3.8 \%$ to close to $40 \%$ (Table 3 ) depending on follow-up duration. All studies mention SP fracture as a subtype of complication.

- The current series identifies older patients at risk for SP fractures; DIAM is contraindicated in these cases.

- Strengths: This study consists of a large number of patients that underwent a specific ISD implantation (DIAM).

- Limitations: Intermediate-term study and lacking longterm outcome.

- Only one ISD device was evaluated, therefore results can only be generalized to this device.

- There was no control group receiving an alternative treatment, so this data does not establish efficacy or comparative safety of ISD compared with alternative treatments.

- A long-term prospective outcome study comparing decompression with decompression with ISD implantation is warranted. 


\section{SUMMARY AND CONCLUSION}

- In most patients who underwent an implantation of DIAM ISD had improved short-term outcome.

- Six percent developed recurrent claudication symptoms in the second-year follow-up, 10.5\% underwent revision surgery.

- In an older population the risk of SP fracture is greater, with failure leading to revision surgery. Other solutions, such as decompression surgery with or without fusion, should be sought for these patients.

\section{REFERENCES}

1. Barbagallo GM, Olindo G, Corbino L, et al (2009) Analysis of complications in patients treated with the X-Stop Interspinous Process Decompression System: proposal for a novel anatomic scoring system for patient selection and review of the literature. Neurosurgery; 65:111-119.

2. Bowers C, Amini A, Dailey AT, et al (2010) Dynamic interspinous process stabilization: review of complications associated with the X-Stop device. Neurosurg Focus; 28:E8.

3. Lindsey DP, Swanson KE, Fuchs P, et al (2003) The effects of an interspinous implant on the kinematics of the instrumented and adjacent levels in the lumbar spine. Spine (Phila Pa 1976); 28:219-217.

4. Mariottini A, Pieri S, Giachi S, et al (2005) Preliminary results of a soft novel lumbar intervertebral prothesis (DIAM) in the degenerative spinal pathology. Acta Neurochir Suppl; 92:129-131.

5. Zucherman JF, Hsu KY, Hartjen CA, et al (2005) A multicenter, prospective, randomized trial evaluating the X STOP interspinous process decompression system for the treatment of neurogenic intermittent claudication: two-year followup results. Spine (Phila Pa 1976); 30:1351-1358.

6. Taylor J, Pupin P, Delajoux S, et al (2007) Device for intervertebral assisted motion: technique and initial results. Neurosurg Focus; 22:E6.

7. Hägg O, Fritzell P, Nordwall A (2003) The clinical importance of changes in outcome scores after treatment for chronic low back pain. Eur Spine J; 12 :12-20.

8. Kondrashov DG, Hannibal M, Hsu KY, et al (2006) Interspinous process decompression with the X-STOP device for lumbar spinal stenosis: a 4-year follow-up study. J Spinal Disord Tech; 19:323-327.

9. Li G, Patil CG, Lad SP, et al (2008) Effects of age and comorbidities on complication rates and adverse outcomes after lumbar laminectomy in elderly patients. Spine (Phila Pa 1976); 33:1250-1255.

\section{EDITORIAL STAFF PERSPECTIVE}

This study on interspinous devices has two shortcomings: it has no control group and it uses a retrospective format for data gathering. There also appeared to have been some selection bias as other interspinous devices were used during the same time frame at the study site. Moreover, the decision to perform formal decompression surgery in addition to placement of the device seems to have been left to surgeon discretion. That stated, the study benefits from a relatively well-sized and followed-up cohort with no reported losses to follow-up. The authors were praised for their diligence in reporting complications and studying their impact on the patients-thus raising the credibility of the study effort.

In the end, the patients seemed to benefit reasonably well from surgeries reported in this series to warrant further investigation and not outrightly condemn the device. Clearly, spinous process fractures in elderly patients with osteopenia seem to be noteworthy in the surgical decision-making process. Based on the vast body of the literature, it appears reasonable to conclude that in suitably selected patients (those without major instability, previous decompression surgery, and with adequate bone stock) it is reasonable to conclude that there appears to be therapeutic equipoise regarding treatment consisting either of decompression and placement of interspinous spacer(s) or suitable decompression surgery alone.

This conclusion leads to the charge for investigators to start formal prospective testing of patients who receive a decompression surgery for stenosis alone or decompression in conjunction with placement of an appropriately selected interspinous spacer following neural element decompression.

Also, the time seems ripe for comparative testing of different devices and their respective complications-for example, rigid devices and those that by virtue of their material design allow for a "cushioning effect" between the spinous processes. 